\title{
Perturbation technique for a class of nonlinear implicit semilinear impulsive integro-differential equations of mixed type with noncompactness measure
}

\author{
Heng-you Lan ${ }^{1,2^{*}}$ and Yi-shun Cui ${ }^{3}$
}

\author{
"Correspondence: \\ hengyoulan@163.com \\ ${ }^{1}$ Institute of Nonlinear Science and \\ Engineering Computing, Sichuan \\ University of Science \& Engineering, \\ Zigong, Sichuan 643000, P.R. China \\ ${ }^{2}$ Key Laboratory of Higher \\ Education of Sichuan Province for \\ Enterprise Informationalization and \\ Internet of Things, Zigong, Sichuan \\ 643000 , P.R. China \\ Full list of author information is \\ available at the end of the article
}

\begin{abstract}
By using the Arzela-Ascoli theorem, the Bellman inequality, and a monotone perturbation iterative technique in the presence of lower and upper solutions, we discuss the existence of mild solutions for a class of nonlinear first-order implicit semilinear impulsive integro-differential equations in Banach spaces. Under wide monotone conditions and the noncompactness measure conditions, we also obtain the existence of extremal solutions and a unique mild solution between lower and upper solutions.
\end{abstract}

Keywords: nonlinear first-order implicit semilinear impulsive integro-differential equation; monotone iterative technique; monotone condition and noncompactness measure condition; lower and upper solution; existence and uniqueness

\section{Introduction}

The theory of impulsive differential equations has become an important area of investigation in recent years stimulated by their numerous applications to problems arising in mechanics, electrical engineering, medicine, biology, ecology, etc. Various evolutionary processes undergo abrupt changes of states at certain moments of time; between intervals of continuous evolution such changes can be well approximated as being instantaneous changes at state, or in the form of impulses. These process are modeled by impulse differential equations and have been the most important research directions and connections for impulsive differential equations; see, for example, [1-7] and the references therein. Subsequently, many authors have investigated the existence of solutions to impulsive differential equations or (implicit) impulsive integro-differential equations with their strong applications in Banach spaces; see [1-27] and the references therein.

Recently, Lan and Cui [15] studied a class of initial value problems of nonlinear firstorder implicit impulsive integro-differential equations in Banach space. By using the Mönch fixed point theorem, they obtained some new existence theorems of solutions for this class of nonlinear first-order implicit impulsive integro-differential equations in $\mathrm{Ba}$ nach spaces under some weaker conditions. Furthermore, some (implicit) impulsive differential equations under various initial and boundary conditions has also been studied by several authors; see, for example, $[11,13,22,24,28]$ and the references therein. By using 
a monotone iterative technique in the presence of lower and upper solutions, Lan [23] discussed the existence of solutions for a new class of nonlinear first-order implicit impulsive integro-differential equations in Banach spaces. Under wide monotone conditions and the noncompactness measure conditions, he also obtained the existence of extremal solutions and a unique solution between lower and upper solutions. In [25], Chen and Li introduced and studied a class of semilinear impulsive evolution equations in Banach spaces by using a mixed monotone iterative technique. The presented results improved and extended some relevant results in ordinary differential equations and partial differential equations. For related works, see $[9,26,27,29,30]$ and the references therein.

On the other hand, the monotone iterative technique, which is one of the approximation methods for finding solutions of a comparatively large class of impulsive differential equations, can be applied in practice easily; see, for example, [14, 16, 17, 23, 25-27, 29]. Further, some nice examples of the monotone iterative technique can be found in [20,21]. As a matter of fact, $\mathrm{Li}$ and $\mathrm{Liu}[16]$ pointed out that 'the monotone iterative technique in the presence of lower and upper solutions is an important method for seeking solutions of differential equations in abstract spaces'. Moreover, Li and Liu [16] used a monotone iterative technique in the presence of lower and upper solutions to discuss the existence of solutions for the initial value problem of the impulsive integro-differential equation of Volterra type in a Banach space. Under monotone conditions and the noncompactness measure condition of the nonlinearity function $f$, the authors also obtained the existence of extremal solutions and a unique solution between lower and upper solutions. In [14], by using a monotone iterative technique in the presence of lower and upper solutions, we discussed the existence of solutions for a new system of nonlinear mixed type implicit impulsive integro-differential equations in Banach spaces. Under some monotonicity conditions and the noncompactness measure conditions, they also obtained the existence of extremal solutions and a unique solution between lower and upper solutions.

Motivated and inspired by the above works, by using the Arzela-Ascoli theorem, the Bellman inequality, and the monotone iterative technique in the presence of lower and upper solutions, we discuss the existence of mild solutions for the following nonlinear first-order implicit semilinear impulsive differential equation problem in Banach space $\mathbb{B}$ : Find $u: J \rightarrow \mathbb{B}$ such that

$$
\left\{\begin{array}{l}
u^{\prime}(t)=A u(t)+f\left(t, u(t), T u(t), u^{\prime}(t)\right), \quad t \neq t_{k} \\
\left.\triangle u\right|_{t=t_{k}}=I_{k}\left(u\left(t_{k}\right)\right), \quad k=1,2, \ldots, m, \\
u\left(t_{0}\right)=u_{0}
\end{array}\right.
$$

where $J=\left[t_{0}, t_{0}+a\right] \subset \mathbb{R}=(-\infty,+\infty)$ is a compact interval, the operator $A$ is the infinitesimal generator of a positive $C_{0}$-semigroup $\left\{G(t), t \geq t_{0}\right\}$ on $\mathbb{B}, f \in C(J \times \mathbb{B} \times \mathbb{B} \times \mathbb{B}, \mathbb{B})$ is a nonlinear continuous operator, $t_{0}<t_{1}<\cdots<t_{m}<t_{0}+a<+\infty, u_{0} \in \mathbb{B}$ is a given element, $\hbar \in C\left(D, \mathbb{R}^{+}\right), D=\{(t, s) \mid s, t \in J, t \geq s\}, \mathbb{R}^{+}=[0,+\infty)$,

$$
T u(t)=\int_{t_{0}}^{t} \hbar(t, s) u(s) d s
$$

and for $k=1,2, \ldots, m, I_{k} \in C[\mathbb{B}, \mathbb{B}]$ is an impulsive function, $\left.\Delta u\right|_{t=t_{k}}$ denotes the jump of $u(t)$ at $t=t_{k}$, i.e., $\left.\Delta u\right|_{t=t_{k}}=u\left(t_{k}^{+}\right)-u\left(t_{k}^{-}\right), u\left(t_{k}^{-}\right)$and $u\left(t_{k}^{+}\right)$represent the left and right limits 
of $u(t)$ at $t=t_{k}$, respectively. Further, under wide monotone conditions and the noncompactness measure conditions, we obtain the existence of extremal solutions and a unique mild solution between lower and upper solutions.

\section{Preliminaries}

Throughout this paper, let $\mathbb{B}$ be an ordered Banach space with the norm $\|\cdot\|$ and partial order $\leq$, whose positive cone $P=\{x \in \mathbb{B} \mid x \geq 0\}$ is normal with normal constant $N$, and $A: \operatorname{dom}(A) \subset \mathbb{B} \rightarrow \mathbb{B}$ be a closed linear operator and generate a $C_{0}$-semigroup $G(t)$ $\left(t \geq t_{0}\right)$ in $\mathbb{B}$. Let $J=\left[t_{0}, t_{0}+a\right], t_{0}<t_{1}<\cdots<t_{m}<t_{0}+a<+\infty, J_{0}=\left[t_{0}, t_{1}\right], J_{1}=\left(t_{1}, t_{2}\right], \ldots$, $J_{k}=\left(t_{k}, t_{k+1}\right], \ldots, J_{m}=\left(t_{m}, t_{0}+a\right]$, and

$$
\begin{aligned}
P C(J, \mathbb{B})= & \left\{x: J \rightarrow \mathbb{B} \mid x(t) \text { is continuous at } t \neq t_{k},\right. \text { and } \\
& \text { left continuous at } \left.t=t_{k}, \text { and } x\left(t_{k}^{+}\right) \text {exists, } k=1,2, \ldots, m\right\} .
\end{aligned}
$$

Evidently, $P C(J, \mathbb{B})$ is a Banach space with norm $\|x\|_{P C}=\sup _{t \in J} x(t)$. Let $J^{\prime}=J \backslash\left\{t_{1}, t_{2}\right.$, $\left.\ldots, t_{m}\right\}$, and $\mathbb{B}_{*}$ be the Banach space generated by $\operatorname{dom}(A)$ with norm $\|\cdot\|_{*}=\|\cdot\|+\|A \cdot\|$. An abstract function $x \in P C(J, \mathbb{B}) \cap C^{1}\left(J^{\prime}, \mathbb{B}\right) \cap C\left(J^{\prime}, \mathbb{B}_{*}\right)$ is called a solution of problem (1.1) if $x(t)$ satisfies all the equalities of (1.1).

Let

$$
P C^{1}(J, \mathbb{B})=\left\{x \in P C(J, \mathbb{B}) \cap C^{1}\left(J^{\prime}, \mathbb{B}\right) \cap C\left(J^{\prime}, \mathbb{B}_{*}\right) \mid x^{\prime}\left(t_{k}^{+}\right), x^{\prime}\left(t_{k}^{-}\right) \text {exist, } k=1,2, \ldots, m\right\},
$$

where $x^{\prime}\left(t_{k}^{+}\right)$and $x^{\prime}\left(t_{k}^{-}\right)$represent the right and left derivatives of $x(t)$ at $t=t_{k}$, respectively. For $x \in P C^{1}(J, \mathbb{B})$, by virtue of the mean value theorem

$$
x\left(t_{k}\right)-x\left(t_{k}-\tau\right) \in \tau \overline{\operatorname{co}}\left\{x^{\prime}(t): t_{k}-\tau<t<t_{k}\right\} \quad(\tau>0),
$$

it is easy to see that the left derivative $x_{-}^{\prime}\left(t_{k}\right)$ exists and

$$
x_{-}^{\prime}\left(t_{k}\right)=\lim _{h \rightarrow 0^{+}} \tau^{-1}\left[x\left(t_{k}\right)-x\left(t_{k}-\tau\right)\right]=x^{\prime}\left(t_{k}^{-}\right)
$$

In the sequel, $x^{\prime}\left(t_{k}\right)$ is understood as $x_{-}^{\prime}\left(t_{k}\right)$, then $x^{\prime} \in P C^{1}(J, \mathbb{B})$. If $x \in P C(J, \mathbb{B}) \cap C^{1}\left(J^{\prime}, \mathbb{B}\right) \cap$ $C\left(J^{\prime}, \mathbb{B}_{*}\right)$ is a solution of problem (1.1), then by the continuity of $f$ and the closed linearity of $A$, we know $x \in P C^{1}(J, \mathbb{B})$. Evidently, $P C^{1}(J, \mathbb{B})$ is a Banach space with norm $\|x\|_{P C^{1}}=$ $\max \left\{\sup _{t \in J}\|x(t)\|, \sup _{t \in J}\left\|x^{\prime}(t)\right\|\right\}$.

A mapping $F: J \rightarrow \mathbb{B}$ is differentiable at $t \in J$ if there exists a $F^{\prime}(t) \in \mathbb{B}$ such that the limits

$$
\lim _{\tau \rightarrow 0^{+}} \frac{F(t+\tau)-F(t)}{\tau} \text { and } \lim _{\tau \rightarrow 0^{+}} \frac{F(t)-F(t-\tau)}{\tau}
$$

exist and are equal to $F^{\prime}(t)$. Here the limits are taken in $\mathbb{B}$. At the endpoints of $J$, we consider the one-sided derivatives.

By the well-known result [31], we know that there exist $C>0$ and $\sigma \in \mathbb{R}$ such that $\|G(t)\| \leq C e^{\sigma t}$. Letting

$$
\delta_{0}:=\inf \left\{\sigma \in \mathbb{B} \mid \exists C>0,\|G(t)\| \leq C e^{\sigma t}\right\},
$$


then $\delta_{0}$ is called the increasing index of $G(t)$. It follows from the properties of the $C_{0}$ semigroup that the $C_{0}$-semigroup $G(t)\left(t \geq t_{0}\right)$ is exponentially stable if and only if $\delta_{0}<0$.

Let $C^{1}(J, \mathbb{B})$ denote the Banach space of all continuous differentiable $\mathbb{B}$-value functions on interval $J$ with norm $\|x\|_{C^{1}}=\max _{t \in J}\left\|x^{\prime}(t)\right\|$. Let $\alpha(\cdot)$ denote the Kuratowski measure of noncompactness of the bounded set. For the details of the definition and properties of the measure of noncompactness, see [32]. For any $E \subset C^{1}(J, \mathbb{B})$ and $t \in J$, set $E(t)=$ $\{x(t) \mid x \in E\} \subset \mathbb{B}$. If $E$ is bounded in $C^{1}(J, \mathbb{B})$, then $E(t)$ is bounded in $\mathbb{B}$, and $\alpha(E(t)) \leq \alpha(E)$.

Lemma 2.1 Assume that the $C_{0}$-semigroup $G(t)\left(t \geq t_{0}\right)$ is exponentially stable, i.e., $\delta_{0}<0$. Then for any $p \in P C^{1}(J, \mathbb{B})$ and $v_{k}, u_{0} \in \mathbb{B}, k=1,2, \ldots, m$, the initial value problem of linear impulsive evolution equation in $\mathbb{B}$

$$
\left\{\begin{array}{l}
u^{\prime}(t)=A u(t)+p(t), \quad t \in J^{\prime}, \\
\left.\Delta u\right|_{t=t_{k}}=v_{k}, \quad k=1,2, \ldots, m, \\
u\left(t_{0}\right)=u_{0},
\end{array}\right.
$$

has a unique mild solution $u \in P C^{1}(J, \mathbb{B})$ expressed by

$$
u(t)=G\left(t-t_{0}\right) u_{0}+\int_{t_{0}}^{t} G(t-s) p(s) d s+\sum_{t_{0}<t_{k}<t} G\left(t-t_{k}\right) v_{k}
$$

Proof It follows from Theorem 2.9 of [31, Chapter 4] and Lemma 2.2 in [26] that this conclusion follows directly.

Lemma 2.2 [33] If $H$ is a bounded subset of $P C^{1}(J, \mathbb{B})$, the element of $H^{\prime}$ is equicontinuous at $J_{k}$ for all $k=0,1,2, \ldots, m$, then

$$
\alpha(H)=\max \left\{\sup _{t \in J} \alpha(H(t)), \sup _{t \in J} \alpha\left(H^{\prime}(t)\right)\right\}
$$

where $H^{\prime}(t)=\left\{x^{\prime}(t): x \in H\right\}$.

Lemma 2.3 [33] Let $E \subset C(J, \mathbb{B})$ be bounded and equicontinuous. Then $\alpha(E(t))$ is continuous on $J$, and

$$
\alpha\left(\left\{\int_{J} x(t) d t \mid x \in E\right\}\right) \leq \int_{J} \alpha(E(t)) d t
$$

Lemma 2.4 [34, Corollary 3.1(b)] Let $E=\left\{x_{n}\right\} \subset P C(J, \mathbb{B})$ be a bounded and countable set. Then $\alpha(E(t))$ is Lebesgue integral on $J$, and

$$
\alpha\left(\left\{\int_{J} x_{n}(t) d t\right\}\right) \leq 2 \int_{J} \alpha(E(t)) d t
$$

\section{Existence and uniqueness theorems}

In this section, we will prove our main results concerning the mild solutions of the nonlinear first-order implicit impulsive integro-differential equation (1.1) in Banach spaces. 
Definition 3.1 If a function $y \in P C^{1}(J, \mathbb{B})$ satisfies

$$
\left\{\begin{array}{l}
y^{\prime}(t) \leq A u(t)+f\left(t, u(t), T u(t), u^{\prime}(t)\right), \quad t \neq t_{k} \\
\left.\triangle y\right|_{t=t_{k}} \leq I_{k}\left(u\left(t_{k}\right)\right), \quad k=1,2, \ldots, m, \\
y\left(t_{0}\right) \leq u_{0}
\end{array}\right.
$$

then we call it a lower solution of problem (1.1); if all the inequalities of (3.1) are inverse, then we call it an upper solution of problem (1.1).

Definition 3.2 A $C_{0}$-semigroup $G(t)\left(t \geq t_{0}\right)$ in $\mathbb{B}$ is said to be positive, if the order inequality $G(t) x \geq \theta$ holds for every $x \geq \theta, x \in \mathbb{B}$, and $t \geq t_{0}$.

It is easy to see that for any $M \geq 0, A-M I$ also generates a $C_{0}$-semigroup $\Gamma(t)=$ $e^{-M(t)} G(t)\left(t \geq t_{0}\right)$ in $\mathbb{B}$. $\Gamma(t)$ is a positive $C_{0}$-semigroup if $G(t)$ is a positive $C_{0}$-semigroup for all $t \geq t_{0}$.

Now, let us first list the following assumptions for convenience:

$\left(\mathrm{H}_{1}\right)$ Problem (1.1) has a lower solution $y_{0} \in P C^{1}(J, \mathbb{B})$ and an upper solution $x_{0} \in P C^{1}(J, \mathbb{B})$ with $y_{0} \leq x_{0}$, and there exist constants $M \in(0,1)$ such that

$$
f\left(t, u_{2}, v_{2}, w_{2}\right)-f\left(t, u_{1}, v_{1}, w_{1}\right) \geq-M\left(u_{2}-u_{1}\right)
$$

for all $t \in J$ and $y_{0}(t) \leq u_{1} \leq u_{2} \leq x_{0}(t), T y_{0}(t) \leq v_{1} \leq v_{2} \leq T x_{0}(t)$, and $y_{0}^{\prime}(t) \leq w_{1} \leq$ $w_{2} \leq x_{0}^{\prime}(t)$.

$\left(\mathrm{H}_{2}\right) I_{k}(x)$ is increasing on the order interval $\left[y_{0}(t), x_{0}(t)\right]$ for $t \in J, k=1,2, \ldots, m$.

$\left(\mathrm{H}_{3}\right)$ There exist $0<2 L<1-M$ such that

$$
\alpha\left(\left\{f\left(t, u_{n}(t), v_{n}(t), w_{n}(t)\right)\right\}\right) \leq L\left[\alpha\left(\left\{u_{n}(t)\right\}\right)+\alpha\left(\left\{v_{n}(t)\right\}\right)+\alpha\left(\left\{w_{n}(t)\right\}\right)\right]
$$

for all $t \in J$, and increasing or decreasing monotonic sequences $\left\{u_{n}\right\} \subset\left[y_{0}(t), x_{0}(t)\right]$, $\left\{v_{n}\right\} \subset\left[T y_{0}(t), T x_{0}(t)\right]$ and $\left\{w_{n}\right\} \subset\left[y_{0}^{\prime}(t), x_{0}^{\prime}(t)\right]$.

In the sequel, we prove the following main results of this paper.

Theorem 3.1 Let $\mathbb{B}$ be an ordered Banach space, whose positive cone $P$ is normal, $A$ : $\operatorname{dom}(A) \subset \mathbb{B} \rightarrow \mathbb{B}$ be a closed linear operator, the positive $C_{0}$-semigroup $G(t)\left(t \geq t_{0}\right)$ generated by $A$ be compact in $\mathbb{B}, f \in C(J \times \mathbb{B} \times \mathbb{B} \times \mathbb{B}, \mathbb{B})$, and $I_{k} \in C(\mathbb{B}, \mathbb{B})$ for $k=1,2, \ldots$, m. Suppose that the conditions $\left(\mathrm{H}_{1}\right) \sim\left(\mathrm{H}_{3}\right)$ hold. Then problem (1.1) has minimal and maximal mild solutions between $\left[y_{0}, x_{0}\right]$, which can be obtained by a monotone iterative procedure starting from $y_{0}$ and $x_{0}$, respectively.

Proof Let $M_{1}=\sup _{t \in J}\|\Gamma(t)\|$ and $M_{2}=\sup _{t \in J}\left\|G^{\prime}(t)\right\|$. For any $u \in P C^{1}(J, \mathbb{B})$, define $F u$ on $J$ by the equation

$$
\begin{aligned}
F u(t)= & \Gamma\left(t-t_{0}\right) u_{0}+\int_{t_{0}}^{t} \Gamma(t-s)\left[f\left(s, u(s), T u(s), u^{\prime}(s)\right)+M u(s)\right] d s \\
& +\sum_{t_{0}<t_{k}<t} \Gamma\left(t-t_{k}\right) I_{k}\left(u\left(t_{k}\right)\right) .
\end{aligned}
$$


It is easy to see that $F: P C^{1}(J, \mathbb{B}) \rightarrow P C^{1}(J, \mathbb{B})$ is continuous. By Lemma 2.1 , we know that the mild solution of problem (1.1) is equivalent to the fixed point of $F$. Since $G(t)$ $\left(t \geq t_{0}\right)$ is a positive $C_{0}$-semigroup, $G(0)=I([31])$ and it follows from assumptions $\left(\mathrm{H}_{1}\right)$ and $\left(\mathrm{H}_{2}\right)$ that $F$ is increasing in $\left[y_{0}, x_{0}\right]$ and maps any bounded set in $\left[y_{0}, x_{0}\right]$ into a bounded set.

We first show that $y_{0} \leq F y_{0}, F x_{0} \leq x_{0}$. Let $p(t)=y_{0}^{\prime}(t)-A y_{0}(t)+M y_{0}(t)$, by the definition of lower solution and (2.1), we know that $p \in P C^{1}(J, \mathbb{B})$ and $p(t) \leq f\left(t, y_{0}(t), T y_{0}(t), y_{0}^{\prime}(t)\right)+$ $M y_{0}(t)$ for $t \in J^{\prime}$. It follows from Lemma 2.1 that

$$
\begin{aligned}
y_{0}(t)= & \Gamma\left(t-t_{0}\right) y_{0}\left(t_{0}\right)+\int_{t_{0}}^{t} \Gamma(t-s) g(s) d s+\left.\sum_{t_{0}<t_{k}<t} \Gamma\left(t-t_{k}\right) \Delta y_{0}\right|_{t=t_{k}} \\
\leq & \Gamma\left(t-t_{0}\right) u_{0}+\int_{t_{0}}^{t} \Gamma(t-s)\left[f\left(t, y_{0}(t), T y_{0}(t), y_{0}^{\prime}(t)\right)+M y_{0}(t)\right] d s \\
& +\sum_{t_{0}<t_{k}<t} \Gamma\left(t-t_{k}\right) I_{k}\left(y_{0}\left(t_{k}\right)\right) \\
= & F y_{0}(t),
\end{aligned}
$$

for all $t \in J$, i.e., $y_{0} \leq F y_{0}$. Similarly, it can be shown that $F x_{0} \leq x_{0}$. Combining these facts and the increasing property of $F$ in $\left[y_{0}, x_{0}\right]$, we see that $F$ maps $\left[y_{0}, x_{0}\right]$ into itself, and $F:\left[y_{0}, x_{0}\right] \rightarrow\left[y_{0}, x_{0}\right]$ is a continuously increasing operator.

Secondly, we prove that $F:\left[y_{0}, x_{0}\right] \rightarrow\left[y_{0}, x_{0}\right]$ is completely continuous. Let

$$
\begin{aligned}
& \Phi u(t)=\int_{t_{0}}^{t} \Gamma(t-s)\left(f\left(s, u(s), T u(s), u^{\prime}(s)\right)+M u(s)\right) d s \\
& \Psi u(t)=\sum_{t_{0}<t_{k}<t} \Gamma\left(t-t_{k}\right) I_{k}\left(u\left(t_{k}\right)\right) .
\end{aligned}
$$

On the one hand, for all $t \in J$, we show that $K(t)=\left\{\Phi u(t) \mid u \in\left[y_{0}, x_{0}\right]\right\}$ is precompact in $\mathbb{B}$. In fact, for any $\epsilon \in\left(t_{0}, t\right)$ and $u \in\left[y_{0}, x_{0}\right]$, it follows from (3.3) that

$$
\begin{aligned}
\Phi_{\epsilon} u(t) & =\int_{t_{0}}^{t-\epsilon} \Gamma(t-s)\left(f\left(s, u(s), T u(s), u^{\prime}(s)\right)+M u(s)\right) d s \\
& =\Gamma(\epsilon) \int_{t_{0}}^{t-\epsilon} \Gamma(t-\epsilon-s)\left(f\left(s, u(s), T u(s), u^{\prime}(s)\right)+M u(s)\right) d s
\end{aligned}
$$

It follows from the condition $\left(\mathrm{H}_{1}\right)$ that

$$
\begin{aligned}
& f\left(t, y_{0}(t), T y_{0}(t), y_{0}^{\prime}(t)\right)+M y_{0}(t) \\
& \quad \leq f\left(t, u(t), T u(t), u^{\prime}(t)\right)+M u(t) \\
& \quad \leq f\left(t, x_{0}(t), T x_{0}(t), x_{0}^{\prime}(t)\right)+M x_{0}(t) .
\end{aligned}
$$

By the normality of the cone $P$, now we know that there exists a constant $M_{3}>0$ such that

$$
\left\|f\left(t, u(t), T u(t), u^{\prime}(t)\right)+M u(t)\right\| \leq M_{3}, \quad \forall u \in\left[y_{0}, x_{0}\right]
$$


From the compactness of $\Gamma(\epsilon)$, we have $K_{\epsilon}(t)=\left\{\Phi_{\epsilon} u(t) \mid u \in\left[y_{0}, x_{0}\right]\right\}$ is precompact in $\mathbb{B}$. Since

$$
\begin{aligned}
\left\|\Phi u(t)-\Phi_{\epsilon} u(t)\right\| & \leq \int_{t-\epsilon}^{t}\|\Gamma(t-s)\| \cdot\left\|f\left(s, u(s), T u(s), u^{\prime}(s)\right)+M u(s)\right\| d s \\
& \leq M_{1} M_{3} \epsilon,
\end{aligned}
$$

the set $K(t)$ is totally bounded in $\mathbb{B}$. Moreover, $K(t)$ is precompact in $\mathbb{B}$.

On the other hand, for all $t_{1}, t_{2} \in J$, from (3.3)-(3.5), we have

$$
\begin{aligned}
\left\|\Phi u\left(t_{2}\right)-\Phi u\left(t_{1}\right)\right\| \leq & \int_{t_{0}}^{t_{2}}\left(\Gamma\left(t_{2}-s\right)-\Gamma\left(t_{1}-s\right)\right)\left(\left\|f\left(s, u(s), T u(s), u^{\prime}(s)\right)+M u(s)\right\|\right) d s \\
& +\int_{t_{1}}^{t_{2}} \Gamma\left(t_{2}-s\right)\left(\left\|f\left(s, u(s), T u(s), u^{\prime}(s)\right)+M u(s)\right\|\right) d s \\
\leq & M_{3} \int_{t_{0}}^{t_{1}}\left\|\Gamma\left(t_{2}-s\right)-\Gamma\left(t_{1}-s\right)\right\| d s+M_{1} M_{3}\left(t_{1}-t_{1}\right) \\
\leq & M_{3} \int_{t_{0}}^{t_{0}+a}\left\|\Gamma\left(t_{2}-t_{1}+s\right)-\Gamma(s)\right\| d s+M_{1} M_{3}\left(t_{1}-t_{1}\right) .
\end{aligned}
$$

The right side of (3.6) relies on $t_{2}-t_{1}$, but it is independent of $u$. Since $G(t)\left(t \geq t_{0}\right)$ is compact, $\Gamma(t)$ is compact and continuous in the uniform operator topology for all $t \geq t_{0}$. Thus, the right side of (3.6) tends to 0 as $t_{2}-t_{1} \rightarrow 0$. Hence, $\Phi\left(\left[y_{0}, x_{0}\right]\right)$ is an equicontinuous function of the cluster in $C^{1}(J, \mathbb{B})$.

Similarly, we can prove the compactness of $\Psi$ in (3.3).

For any $t \in J$, since $\left\{F u(t) \mid u \in\left[y_{0}, x_{0}\right]\right\}=\left\{\Gamma\left(t-t_{0}\right)+\Phi u(t)+\Psi u(t) \mid u \in\left[y_{0}, x_{0}\right]\right\}$, and $F u\left(t_{0}\right)=u_{0}$ is precompact in $\mathbb{B}$, we know that $F\left(\left[y_{0}, x_{0}\right]\right)$ is precompact in $C^{1}\left(J_{k}, \mathbb{B}\right)$ by using the Arzela-Ascoli theorem. Thus, $F:\left[y_{0}, x_{0}\right] \rightarrow\left[y_{0}, x_{0}\right]$ is completely continuous.

Finally, we show that problem (1.1) has minimal and maximal mild solutions between $\left[y_{0}, x_{0}\right]$, which can be obtained by a monotone iterative procedure starting from $y_{0}$ and $x_{0}$, respectively.

It follows from the completely continuity of $F$ that $F$ has minimal and maximal fixed points $\underline{u}$ and $\bar{u}$ in $\left[y_{0}, x_{0}\right]$, and so they are the minimal and maximal mild solutions of problem (1.1) in $\left[y_{0}, x_{0}\right]$, respectively.

On the other hand, from the above discussions, we know that $F:\left[y_{0}, x_{0}\right] \rightarrow\left[y_{0}, x_{0}\right]$ is a continuously increasing operator. Now, we define two sequences $\left\{y_{n}\right\}$ and $\left\{x_{n}\right\}$ in $\left[y_{0}, x_{0}\right]$ by the iterative scheme

$$
y_{n}=F y_{n-1}, \quad x_{n}=F x_{n-1}, \quad n=1,2, \ldots
$$

Then it follows from the monotonicity of $F$ that

$$
y_{0} \leq y_{1} \leq \cdots \leq y_{n} \leq \cdots \leq x_{n} \leq \cdots \leq x_{1} \leq x_{0} .
$$

We prove that $\left\{y_{n}\right\}$ and $\left\{x_{n}\right\}$ are uniformly convergent in $J$.

For convenience, let $E=\left\{y_{n} \mid n \in \mathbb{N}\right\}$ and $E_{0}=\left\{y_{n-1} \mid n \in \mathbb{N}\right\}$. Since $E=F\left(E_{0}\right)$, by (3.2) and the boundedness of $E_{0}$, we easily see that $E$ is equicontinuous in every interval $J_{k}^{\prime}$, where 
$J_{1}^{\prime}=\left[t_{0}, t_{1}\right]$ and $J_{k}^{\prime}=\left(t_{k-1}, t_{k}\right], k=2,3, \ldots, m$. From $E_{0}=E \cup\left\{y_{0}\right\}$ and Lemma 2.2, it follows that $\alpha\left(E_{0}(t)\right)=\alpha(E(t))$ and

$$
\alpha^{1}(E(t))=\max \left\{\alpha(E(t)), \alpha\left(E^{\prime}(t)\right)\right\}
$$

for all $t \in J$. Letting

$$
\phi(t)=\alpha^{1}(E(t))=\alpha^{1}\left(E_{0}(t)\right), \quad t \in J
$$

by Lemma 2.3 , we know that $\phi \in P C^{1}\left(J, \mathbb{R}^{+}\right)$. Going from $J_{1}^{\prime}$ to $J_{m+1}^{\prime}$ interval by interval, we show that $\phi(t) \equiv 0$ in $J$.

In fact, for $t \in J$, there exists a $J_{k}^{\prime}$ such that $t \in J_{k}^{\prime}$. By Lemma 2.3, we have

$$
\begin{aligned}
\alpha\left(T\left(E_{0}(t)\right)\right)= & \alpha\left(\left\{\int_{t_{0}}^{t} \hbar(t, s) y_{n-1}(s) d s \mid n \in \mathbb{N}\right\}\right) \\
\leq & \sum_{j=1}^{k-1} \alpha\left(\left\{\int_{t_{j-1}}^{t_{j}} \hbar(t, s) y_{n-1}(s) d s \mid n \in \mathbb{N}\right\}\right) \\
& +\alpha\left(\left\{\int_{t_{k-1}}^{t} \hbar(t, s) y_{n-1}(s) d s \mid n \in \mathbb{N}\right\}\right) \\
\leq & \hbar_{0} \sum_{j=1}^{k-1} \int_{t_{j-1}}^{t_{j}} \alpha\left(E_{0}(s)\right) d s+\hbar_{0} \int_{t_{k-1}}^{t} \alpha\left(E_{0}(s)\right) d s \\
\leq & \hbar_{0} \sum_{j=1}^{k-1} \int_{t_{j-1}}^{t_{j}} \phi(s) d s+\hbar_{0} \int_{t_{k-1}}^{t} \phi(s) d s \\
= & \hbar_{0} \int_{t_{0}}^{t} \phi(s) d s,
\end{aligned}
$$

where $\hbar_{0}=\max \{|\hbar(t, s)|:(t, s) \in D\}$. Hence,

$$
\int_{t_{0}}^{t} \alpha\left(T\left(E_{0}(s)\right)\right) d s \leq a \hbar_{0} \int_{t_{0}}^{t} \phi(s) d s
$$

It follows from (3.2), Lemma 2.4, assumption $\left(\mathrm{H}_{3}\right)$ and (3.9) that, for $t \in J_{1}^{\prime}$,

$$
\begin{aligned}
\alpha(E(t)) & =\alpha\left(F\left(E_{0}(t)\right)\right) \\
& =\alpha\left(\left\{\int_{t_{0}}^{t} \Gamma(t-s)\left(f\left(s, y_{n-1}(s), T y_{n-1}(s), y_{n-1}^{\prime}(s)\right)+M y_{n-1}(s)\right) d s \mid n \in \mathbb{N}\right\}\right) \\
& \leq 2 \int_{t_{0}}^{t} \Gamma(t-s) \alpha\left(\left(\left(f\left(s, y_{n-1}(s), T y_{n-1}(s), y_{n-1}^{\prime}(s)\right)+M y_{n-1}(s)\right) \mid n \in \mathbb{N}\right)\right) d s \\
& \leq 2 M_{1} \int_{t_{0}}^{t}\left\{L\left[\alpha\left(E_{0}(s)\right)+\alpha\left(T\left(E_{0}(s)\right)\right)+\alpha\left(E_{0}^{\prime}(s)\right)\right]+M \alpha\left(E_{0}(s)\right)\right\} d s \\
& \leq 2 M_{1}\left[(L+M) \int_{t_{0}}^{t} \alpha\left(E_{0}(s)\right) d s+L \int_{t_{0}}^{t} \alpha\left(T\left(E_{0}(s)\right)\right) d s+L \int_{t_{0}}^{t} \alpha\left(E_{0}^{\prime}(s)\right) d s\right] \\
& \leq 2 M_{1}\left(M+a \hbar_{0} L+2 L\right) \int_{t_{0}}^{t} \phi(s) d s,
\end{aligned}
$$




$$
\begin{aligned}
\alpha\left(E^{\prime}(t)\right)= & \alpha\left(\left(F E_{0}\right)^{\prime}(t)\right) \\
= & \alpha\left(\frac{d}{d t}\left\{\int_{t_{0}}^{t} \Gamma(t-s)\left(f\left(s, y_{n-1}(s), T y_{n-1}(s), y_{n-1}^{\prime}(s)\right)+M y_{n-1}(s)\right) d s \mid n \in \mathbb{N}\right\}\right) \\
= & \alpha\left(\left\{\int_{t_{0}}^{t} \Gamma^{\prime}(t-s) f\left(s, y_{n-1}(s), T y_{n-1}(s), y_{n-1}^{\prime}(s)\right) d s\right.\right. \\
& \left.\left.+M y_{n-1}(t)+\Gamma(0) f\left(t, y_{n-1}(t), T y_{n-1}(t), y_{n-1}^{\prime}(t)\right) \mid n \in \mathbb{N}\right\}\right) \\
= & \alpha\left(\left\{-M F\left(y_{n-1}(t)\right)\right.\right. \\
& +\int_{t_{0}}^{t} G^{\prime}(t-s) e^{-M(t-s)} f\left(s, y_{n-1}(s), T y_{n-1}(s), y_{n-1}^{\prime}(s)\right) d s \\
& \left.\left.+M y_{n-1}(t)+G(0) f\left(t, y_{n-1}(t), T y_{n-1}(t), y_{n-1}^{\prime}(t)\right) \mid n \in \mathbb{N}\right\}\right) \\
\leq & -M \alpha\left(F\left(E_{0}(t)\right)\right) \\
& +\alpha\left(\int_{t_{0}}^{t} G^{\prime}(t-s) e^{-M(t-s)} f\left(s, y_{n-1}(s), T y_{n-1}(s), y_{n-1}^{\prime}(s)\right) d s \mid n \in \mathbb{N}\right) \\
& +M \alpha\left(E_{0}(t)\right)+\alpha\left(\left\{f\left(t, y_{n-1}(t s), T y_{n-1}(t), y_{n-1}^{\prime}(t)\right)\right\}\right) \\
\leq & -M \alpha\left(F\left(E_{0}(t)\right)\right) \\
& +2 \int_{t_{0}}^{t} G^{\prime}(t-s) e^{-M(t-s)} \alpha\left(f\left(s, y_{n-1}(s), T y_{n-1}(s), y_{n-1}^{\prime}(s)\right) \mid n \in \mathbb{N}\right) d s \\
& +M \alpha\left(E_{0}(t)\right)+\alpha\left(\left\{f\left(t, y_{n-1}(t s), T y_{n-1}(t), y_{n-1}^{\prime}(t)\right)\right\}\right) \\
\leq & -M \alpha\left(F\left(E_{0}(t)\right)\right)+M \alpha\left(E_{0}(t)\right)+\zeta\left[\alpha\left(E_{0}(t)\right)+\alpha\left(T\left(E_{0}\right)(t)\right)+\alpha\left(E_{0}^{\prime}(t)\right)\right] \\
\leq & {\left[a \hbar_{0} \zeta-2 M\left(M+a \hbar_{0} \zeta+2 \zeta\right)\right] \int_{t_{0}}^{t} \phi(s) d s+(\zeta+M) \alpha\left(E_{0}(t)\right)+L \alpha\left(E_{0}^{\prime}(t)\right) } \\
\leq & {\left[a \hbar_{0} \zeta-2 M\left(M+a \hbar_{0} \zeta+2 \zeta\right)\right] \int_{t_{0}}^{t} \phi(s) d s+(M+2 \zeta) \alpha^{1}\left(E_{0}(t)\right) } \\
& \\
& \\
&
\end{aligned}
$$

and so

$$
\phi(t) \leq \Gamma \int_{t_{0}}^{t} \phi(s) d s+(M+2 L) \phi(t),
$$

i.e.,

$$
\phi(t) \leq \Theta \int_{t_{0}}^{t} \phi(s) d s
$$

where $\zeta=L\left(1+2 a M_{2}\right), \Gamma=\max \left\{2\left(M+a \hbar_{0} L+2 L\right), a \hbar_{0} \zeta-2 M\left(M+a \hbar_{0} \zeta+2 \zeta\right)\right\}$, and $\Theta=\Gamma /(1-M-2 \zeta)$. Hence, by the Bellman inequality, we know that $\phi(t) \equiv 0$ in $J_{1}^{\prime}$. In particular, $\alpha^{1}\left(E\left(t_{1}\right)\right)=\alpha^{1}\left(E_{0}\left(t_{1}\right)\right)=\phi\left(t_{1}\right)=0$, and so $\alpha\left(E\left(t_{1}\right)\right)=\alpha\left(E_{0}\left(t_{1}\right)\right)=0$, this means that $E\left(t_{1}\right)$ and $E_{0}\left(t_{1}\right)$ are precompact in $\mathbb{B}$. Thus $I_{1}\left(E_{0}\left(t_{1}\right)\right)$ is precompact in $\mathbb{B}$, and $\alpha\left(I_{1}\left(E_{0}\left(t_{1}\right)\right)\right)=0$. 
Now, for $t \in J_{2}^{\prime}$, by (3.2) and the above argument for $J_{1}^{\prime}$, we have

$$
\begin{aligned}
\alpha(E(t))= & \alpha(E(t))=\alpha\left(F\left(E_{0}\right)(t)\right) \\
= & \alpha\left(\left\{\Gamma\left(t-t_{0}\right) u_{0}+\int_{t_{0}}^{t} \Gamma(t-s)\left(f\left(s, y_{n-1}(s), T y_{n-1}(s), y_{n-1}^{\prime}(s)\right)+M y_{n-1}(s)\right) d s\right.\right. \\
& \left.\left.+\Gamma\left(t-t_{1}\right) I_{1}\left(y_{n-1}\left(t_{1}\right)\right) \mid n \in \mathbb{N}\right\}\right) \\
\leq & 2\left(L+M+a \hbar_{0} L\right) \int_{t_{0}}^{t} \phi(s) d s+\alpha\left(I_{1}\left(E_{0}\left(t_{1}\right)\right)\right) \\
\leq & 2\left(M+a \hbar_{0} L+2 L\right) \int_{t_{0}}^{t} \phi(s) d s \\
= & 2\left(M+a \hbar_{0} L+2 L\right) \int_{t_{1}}^{t} \phi(s) d s
\end{aligned}
$$

and

$$
\phi(t) \leq \Theta \int_{t_{1}}^{t} \phi(s) d s
$$

Again by the Bellman inequality, we know that $\phi(t) \equiv 0$ in $J_{2}^{\prime}$, from which we obtain $\alpha\left(E_{0}\left(t_{2}\right)\right)=0$ and $\alpha\left(I_{2}\left(E_{0}\left(t_{2}\right)\right)\right)=0$.

Continuing such a process interval by interval up to $J_{m+1}^{\prime}$, we can prove that $\phi(t) \equiv 0$ in every $J_{k}^{\prime}, k=1,2, \ldots, m+1$.

For any $J_{k}$, if we modify the value of $y_{n}$ at $t=t_{k-1}$ via $y_{n}\left(t_{k-1}\right)=y_{n}\left(t_{k-1}^{+}\right), n \in \mathbb{N}$, then $\left\{y_{n}\right\} \subset C^{1}\left(J_{k}, \mathbb{B}\right)$ and it is equicontinuous. Since $\alpha\left(\left\{y_{n}(t)\right\}\right) \equiv 0,\left\{y_{n}(t)\right\}$ is precompact in $\mathbb{B}$ for every $t \in J_{k}$. By the Arzela-Ascoli theorem, we know that $\left\{y_{n}\right\}$ is precompact in $C^{1}\left(J_{k}, \mathbb{B}\right)$. Hence, $\left\{y_{n}\right\}$ has a convergent subsequence in $C^{1}\left(J_{k}, \mathbb{B}\right)$. Combining this with the monotonicity (3.8), we easily prove that $\left\{y_{n}\right\}$ itself is convergent in $C^{1}\left(J_{k}, \mathbb{B}\right)$. In particular, $\left\{y_{n}(t)\right\}$ is uniformly convergent in $J_{k}^{\prime}$. Consequently, $\left\{y_{n}(t)\right\}$ is uniformly convergent over the whole of $J$.

Using a similar argument to that for $\left\{y_{n}(t)\right\}$, we can prove that $\left\{x_{n}(t)\right\}$ is also uniformly convergent in $J$. Hence, $\left\{y_{n}(t)\right\}$ and $\left\{x_{n}(t)\right\}$ are convergent in $P C^{1}(J, \mathbb{B})$. Setting

$$
\underline{u}=\lim _{n \rightarrow \infty} y_{n}, \quad \bar{u}=\lim _{n \rightarrow \infty} x_{n} \quad \text { in } P C^{1}(J, \mathbb{B})
$$

and $n \rightarrow \infty$ in (3.7) and (3.8), then we have $v_{0} \leq \underline{u} \leq \bar{u} \leq x_{0}$ and

$$
\underline{u}=F \underline{u}, \quad \bar{u}=F \bar{u} .
$$

By the monotonicity of $F$, it is easy to see that $\underline{u}$ and $\bar{u}$ are the minimal and maximal fixed points of $F$ in $\left[y_{0}, x_{0}\right]$, and therefore they are the minimal and maximal mild solutions of problem (1.1) in $\left[y_{0}, x_{0}\right]$, respectively. This completes the proof.

Remark 3.1 In Theorem 3.1, if $\mathbb{B}$ is weakly sequentially complete, the condition $\left(\mathrm{H}_{3}\right)$ holds automatically. In fact, by Theorem 2.2 of [35], any monotonic and order-bounded sequence is precompact. Let $\left\{x_{n}\right\}$ and $\left\{y_{n}\right\}$ be two increasing or decreasing sequences 
obeying condition $\left(\mathrm{H}_{3}\right)$, then, by condition $\left(\mathrm{H}_{1}\right),\left\{f\left(t, x_{n}, y_{n}, z_{n}\right)+M x_{n}\right\}$ is a monotonic and order-bounded sequence. By the property of the measure of noncompactness, we have

$$
\begin{aligned}
& \alpha\left(\left\{A x_{n}(t)+f\left(t, x_{n}, y_{n}, z_{n}\right)+M x_{n}\right\}\right) \\
& \quad \leq \alpha\left(\left\{A x_{n}(t)+f\left(t, x_{n}, y_{n}, z_{n}\right)+M x_{n}\right\}\right)+M \alpha\left(\left\{x_{n}\right\}\right)=0 .
\end{aligned}
$$

Hence, condition $\left(\mathrm{H}_{3}\right)$ holds.

From Theorem 3.1, we obtain the following result.

Corollary 3.1 Let $\mathbb{B}$ be an ordered and weakly sequentially complete Banach space, whose positive cone $P$ is normal, $f \in C(J \times \mathbb{B} \times \mathbb{B} \times \mathbb{B}, \mathbb{B})$, and $I_{k} \in C(\mathbb{B}, \mathbb{B}), k=1,2, \ldots, m$. If the conditions $\left(\mathrm{H}_{1}\right)$ and $\left(\mathrm{H}_{2}\right)$ are satisfied, then problem (1.1) has minimal and maximal mild solutions between $y_{0}$ and $x_{0}$, which can be obtained by a monotone iterative procedure starting from $y_{0}$ and $x_{0}$, respectively.

Next we discuss the uniqueness of the mild solution to problem (1.1) in $\left[y_{0}, x_{0}\right]$. Assume we replace the assumption $\left(\mathrm{H}_{3}\right)$ by the following assumption.

$\left(\mathrm{H}_{4}\right)$ There exist positive constants $C_{i}(i=1,2,3)$ with $C_{3}<1$ such that

$$
\begin{aligned}
& f\left(t, u_{2}, v_{2}, w_{2}\right)-f\left(t, u_{1}, v_{1}, w_{1}\right) \leq C_{1}\left(u_{2}-u_{1}\right)+C_{2}\left(v_{2}-v_{1}\right)+C_{3}\left(w_{2}-w_{1}\right), \\
& \forall t \in J, \quad y_{0}(t) \leq u_{1} \leq u_{2} \leq x_{0}(t), \quad T y_{0}(t) \leq y_{1} \leq y_{2} \leq T x_{0}(t),
\end{aligned}
$$

for all $t \in J$ and $y_{0}(t) \leq u_{1} \leq u_{2} \leq x_{0}(t), \lambda_{1} T y_{0}(t) \leq v_{1} \leq v_{2} \leq \lambda_{1} T x_{0}(t)$ and $\lambda_{2} y_{0}^{\prime}(t) \leq w_{1} \leq$ $w_{2} \leq \lambda_{2} x_{0}^{\prime}(t)$. Then we have the following unique existence result.

Theorem 3.2 Let $\mathbb{B}$ be an ordered Banach space, whose positive cone $P$ is normal, $f \in$ $C(J \times \mathbb{B} \times \mathbb{B} \times \mathbb{B}, \mathbb{B})$ and $I_{k} \in C(\mathbb{B}, \mathbb{B}), k=1,2, \ldots, m$. If the conditions $\left(\mathrm{H}_{1}\right),\left(\mathrm{H}_{2}\right)$, and $\left(\mathrm{H}_{4}\right)$ hold, then problem (1.1) has a unique mild solution between $y_{0}$ and $x_{0}$, which can be obtained by a monotone iterative procedure starting from $y_{0}$ or $x_{0}$.

Proof We first prove that $\left(\mathrm{H}_{1}\right)$ and $\left(\mathrm{H}_{4}\right)$ imply $\left(\mathrm{H}_{3}\right)$. In fact, for $t \in J$, let $\left\{u_{n}\right\} \subset\left[y_{0}(t), x_{0}(t)\right]$, $\left\{v_{n}\right\} \subset\left[T y_{0}(t), T x_{0}(t)\right]$, and $\left\{w_{n}\right\} \subset\left[y_{0}^{\prime}(t), x_{0}^{\prime}(t)\right]$ be increasing sequences. For $m, n \in \mathbb{N}$ with $m>n$, by $\left(\mathrm{H}_{1}\right)$ and $\left(\mathrm{H}_{4}\right)$,

$$
\begin{aligned}
\theta & \leq\left(f\left(t, u_{m}, v_{m}, w_{m}\right)-f\left(t, u_{n}, v_{n}, w_{n}\right)\right)+M\left(u_{m}-u_{n}\right) \\
& \leq\left(C_{1}+M\right)\left(u_{m}-u_{n}\right)+C_{2}\left(v_{m}-v_{n}\right)+C_{3}\left(w_{m}-w_{n}\right) .
\end{aligned}
$$

By this inequality and the normality of cone $P$, we have

$$
\begin{aligned}
& \left\|f\left(t, u_{m}, v_{m}, w_{m}\right)-f\left(t, u_{n}, v_{n}, w_{n}\right)\right\| \\
& \quad \leq N\left\|\left(C_{1}+M\right)\left(u_{m}-u_{n}\right)+C_{2}\left(v_{m}-v_{n}\right)+C_{3}\left(w_{m}-w_{n}\right)\right\|+M\left\|u_{m}-u_{n}\right\| \\
& \quad \leq\left(M+M N+N C_{1}\right)\left\|u_{m}-u_{n}\right\|+N C_{2}\left\|v_{m}-v_{n}\right\|+N C_{3}\left(w_{m}-w_{n}\right) .
\end{aligned}
$$


From this inequality and the definition of the measure of noncompactness, it follows that

$$
\begin{aligned}
& \alpha\left(\left\{f\left(t, u_{n}, v_{n}, w_{n}\right)\right\}\right) \\
& \quad \leq\left(M+M N+N C_{1}\right) \alpha\left(\left\{u_{n}\right\}\right)+N C_{2} \alpha\left(\left\{v_{n}\right\}\right)+N C_{3} \alpha\left(\left\{w_{n}\right\}\right) \\
& \quad \leq L^{\prime}\left[\alpha\left(\left\{u_{n}\right\}\right)+\alpha\left(\left\{v_{n}\right\}\right)+\alpha\left(\left\{w_{n}\right\}\right)\right],
\end{aligned}
$$

where $L^{\prime}=\max \left\{M+N M+N C_{1}, N C_{2}, N C_{3}\right\}$. If $\left\{u_{n}\right\},\left\{v_{n}\right\}$, and $\left\{w_{n}\right\}$ are decreasing sequences, the above inequality is also valid. Hence $\left(\mathrm{H}_{3}\right)$ holds.

Therefore, by Theorem 3.1, problem (1.1) has a minimal solution $\underline{u}$ and a maximal solution $\bar{u}$ in $\left[y_{0}, x_{0}\right]$. By the proof of Theorem 3.1, (3.7), (3.8), (3.10), and (3.11) are valid. Going from $J_{1}^{\prime}$ to $J_{m+1}^{\prime}$ interval by interval, we show that $\underline{u}(t) \equiv \bar{u}(t)$ in every $J_{k}^{\prime}, k=1,2, \ldots, m+1$. Indeed, for $t \in J_{1}^{\prime}$, by (3.11) and (3.2) and assumption $\left(\mathrm{H}_{4}\right)$, we have

$$
\begin{aligned}
\theta \leq & \bar{u}(t)-\underline{u}(t)=F \bar{u}(t)-F \underline{u}(t) \\
= & \int_{t_{0}}^{t} \Gamma(t-s)\left[f\left(s, \bar{u}(s), T \bar{u}(s), \bar{u}^{\prime}(s)\right)-f\left(s, \underline{u}(s), T \underline{u}(s), \underline{u}^{\prime}(s)\right)+M(\bar{u}(s)-\underline{u}(s))\right] d s \\
\leq & \int_{t_{0}}^{t} M_{1}\left[\left(M+C_{1}\right)(\bar{u}(s)-\underline{u}(s))+C_{2}(T \bar{u}(s)-T \underline{u}(s))+C_{3}\left(\bar{u}^{\prime}(s)-\underline{u}^{\prime}(s)\right)\right] d s \\
\leq & M_{1}\left(M+C_{1}\right) \int_{t_{0}}^{t}(\bar{u}(s)-\underline{u}(s)) d s \\
& +M_{1} C_{2} \hbar_{0} \int_{t_{0}}^{t} \int_{t_{0}}^{s}(\bar{u}(\tau)-\underline{u}(\tau)) d \tau d s+M_{1} C_{3}(\bar{u}(t)-\underline{u}(t)) \\
& \leq M_{1}\left(M+C_{1}+a C_{2} \hbar_{0}\right) \int_{t_{0}}^{t}(\bar{u}(s)-\underline{u}(s)) d s+M_{1} C_{3}(\bar{u}(t)-\underline{u}(t)),
\end{aligned}
$$

where $M_{1}=\sup _{t \in J}\|\Gamma(t)\|$. It follows from (3.12) and the normality of cone $P$ that

$$
\|\bar{u}(t)-\underline{u}(t)\| \leq M_{1} N\left(M+C_{1}+a C_{2} \hbar_{0}\right) \int_{t_{0}}^{t}\|\bar{u}(s)-\underline{u}(s)\| d s+M_{1} C_{3}\|\bar{u}(t)-\underline{u}(t)\|,
$$

i.e.,

$$
\|\bar{u}(t)-\underline{u}(t)\| \leq \frac{M_{1} N\left(M+C_{1}+a C_{2} \hbar_{0}\right)}{1-M_{1} C_{3}} \int_{t_{0}}^{t}\|\bar{u}(s)-\underline{u}(s)\| d s
$$

Thus, by the Bellman inequality, we obtain $\underline{u}(t) \equiv \bar{u}(t)$ in $J_{1}^{\prime}$.

For $t \in J_{2}^{\prime}$, since $I_{1}\left(\bar{u}\left(t_{1}\right)\right)=I_{1}\left(\underline{u}\left(t_{1}\right)\right)$, using (3.2) and by completely the same argument as above for $t \in J_{1}^{\prime}$, we can prove that

$$
\begin{aligned}
\|\bar{u}(t)-\underline{u}(t)\| & \leq \frac{M_{1} N\left(M+C_{1}+a C_{2} \hbar_{0}\right)}{1-M_{1} C_{3}} \int_{t_{0}}^{t}\|\bar{u}(s)-\underline{u}(s)\| d s \\
& =\frac{M_{1} N\left(M+C_{1}+a C_{2} \hbar_{0}\right)}{1-M_{1} C_{3}} \int_{t_{1}}^{t}\|\bar{u}(s)-\underline{u}(s)\| d s .
\end{aligned}
$$

Again, by the Bellman inequality, we obtain $\underline{u}(t) \equiv \bar{u}(t)$ in $J_{2}^{\prime}$. 
Continuing such a process interval by interval up to $J_{m+1}^{\prime}$, we see that $\underline{u}(t) \equiv \bar{u}(t)$ over the whole of $J$. Hence, $u^{*}:=\underline{u}=\bar{u}$ is the unique mild solution of problem (1.1) in $\left[y_{0}, x_{0}\right]$, which can be obtained by the monotone iterative procedure (3.7) starting from $y_{0}$ or $x_{0}$.

Remark 3.2 (1) Using the above argument method interval by interval from $J_{1}^{\prime}$ to $J_{m+1}^{\prime}$, we can also improve the main results in [19] and [21], and delete some restrictive conditions there.

(2) In this study, the equicontinuity of the semigroup $G(t)\left(t \geq t_{0}\right)$ generated by $A$ is not required.

\section{Concluding remarks}

In this paper, we introduce and study the following nonlinear first-order implicit impulsive differential equation problem in Banach space $\mathbb{B}$ :

Find $u: J \rightarrow \mathbb{B} \times \mathbb{B} \times \mathbb{B}$ such that

$$
\left\{\begin{array}{l}
u^{\prime}(t)=A u(t)+f\left(t, u(t), T u(t), u^{\prime}(t)\right), \quad t \neq t_{k}, \\
\left.\triangle u\right|_{t=t_{k}}=I_{k}\left(u\left(t_{k}\right)\right), \quad k=1,2, \ldots, m, \\
u\left(t_{0}\right)=u_{0} .
\end{array}\right.
$$

By using a monotone iterative technique in the presence of lower and upper solutions, the existence of extremal solutions and a unique mild solution between the lower and upper solutions are obtained under wide monotone conditions and the noncompactness measure conditions. The results presented in this paper improved and generalized some known results concerned with the integro-differential equations and classical (abstract) differential equations.

Moreover, we remark that if the lower solution and the upper solution for problem (1.1) do not exist, then we have the following results.

Theorem 4.1 Let $\mathbb{B}$ be an ordered Banach space, whose positive cone $P$ is normal, $A$ : $\operatorname{dom}(A) \subset \mathbb{B} \rightarrow \mathbb{B}$ be a closed linear operator and generate a positive $C_{0}$-semigroup $G(t)$ $\left(t \geq t_{0}\right)$ in $\mathbb{B}, f \in C(J \times \mathbb{B} \times \mathbb{B} \times \mathbb{B}, \mathbb{B})$, and $I_{k} \in C(\mathbb{B}, \mathbb{B}), k=1,2, \ldots, m$. Assume that there exist $b>0, x_{0} \in \operatorname{dom}(A), x_{0} \geq \theta, y_{k} \in \operatorname{dom}(A), y_{k} \geq \theta, k=1,2, \ldots, m, h \in P C^{1}(J, \mathbb{B})$, and $h(t) \geq \theta$ such that

$$
\begin{array}{ll}
f\left(t, x, T x, x^{\prime}\right) \leq b x+h(t), & I_{k}(x) \leq y_{k}, \quad x \geq \theta ; \\
f\left(t, x, T x, x^{\prime}\right) \geq b x-h(t), & I_{k}(x) \geq-y_{k}, \quad x \leq \theta .
\end{array}
$$

Then the following results hold:

(1) If the $C_{0}$-semigroup $G(t)\left(t \geq t_{0}\right)$ is compact in $\mathbb{B}$, and the conditions $\left(\mathrm{H}_{1}\right)$ and $\left(\mathrm{H}_{2}\right)$ in Section 3 are satisfied, then problem (1.1) has minimal and maximal mild solutions.

(2) Problem (1.1) has minimal and maximal mild solutions when the conditions $\left(\mathrm{H}_{1}\right) \sim\left(\mathrm{H}_{3}\right)$ in Section 3 are satisfied.

(3) If the positive cone $P$ is regular, and the conditions $\left(\mathrm{H}_{1}\right)$ and $\left(\mathrm{H}_{2}\right)$ in Section 3 are satisfied, then problem (1.1) has minimal and maximal mild solutions. 
(4) Problem (1.1) has a unique mild solution when the conditions $\left(\mathrm{H}_{1}\right),\left(\mathrm{H}_{2}\right)$, and $\left(\mathrm{H}_{4}\right)$ in Section 3 are satisfied.

Proof Firstly, we consider the following initial value problem of the linear impulsive evolution equation in $\mathbb{B}$ :

$$
\left\{\begin{array}{l}
u^{\prime}(t)=A u(t)+b u(t)+h(t), \quad t \in J^{\prime} \\
\left.\triangle u\right|_{t=t_{k}}=y_{k}, \quad k=1,2, \ldots, m, \\
u\left(t_{0}\right)=x_{0} .
\end{array}\right.
$$

Since $(A+b I)$ generates a positive $C_{0}$-semigroup $\Gamma(t)=e^{b t} G(t)(t \geq 0)$ in $\mathbb{B}$, it follows from Theorem 2.9 in [31, Chapter 4] and Lemma 2.1, that problem (4.1) has a unique positive classical solution $\hat{u} \in P C^{1}(J, E)$. Let $y_{0}=-\hat{u}, x_{0}=\hat{u}$, it is easy to see that $y_{0}$ and $x_{0}$ are the lower solution and the upper solution of problem (1.1), respectively. So, our conclusions (1)-(4) follow from Theorems 3.1 and 3.2.

\section{Competing interests}

The authors declare that they have no competing interests.

\section{Authors' contributions}

$\mathrm{H}-\mathrm{YL}$ conceived of the study and participated in its design and coordination. Y-SC carried out the proof of the corollaries and gave some remarks to show the main results. All authors read and approved the final manuscript.

\section{Author details}

${ }^{1}$ Institute of Nonlinear Science and Engineering Computing, Sichuan University of Science \& Engineering, Zigong Sichuan 643000, P.R. China. ${ }^{2}$ Key Laboratory of Higher Education of Sichuan Province for Enterprise Informationalization and Internet of Things, Zigong, Sichuan 643000, P.R. China. ${ }^{3}$ College of Materials and Chemical Engineering, Sichuan University of Sciences \& Engineering, Zigong, Sichuan 643000, P.R. China.

\section{Acknowledgements}

We thank the referees' valuable comments and suggestions to improve our paper.

This work was partially supported by Sichuan Province Cultivation Fund Project of Academic and Technical Leaders, and the Open Research Fund of Key Laboratory of Higher Education of Sichuan Province for Enterprise Informationalization and Internet of Things (2013WZJ01)

Received: 27 August 2014 Accepted: 19 December 2014 Published online: 30 January 2015

\section{References}

1. Samoilenko, AM, Perestyuk, NA: Impulsive Differential Equations. World Scientific, Singapore (1995)

2. Cuevas, C, N'Guérékata, GM, Rabelo, M: Mild solutions for impulsive neutral functional differential equations with state-dependent delay. Semigroup Forum 80(3), 375-390 (2010)

3. Cuevas, C, Hernández, E, Rabelo, M: The existence of solutions for impulsive neutral functional differential equations. Comput. Math. Appl. 58(4), 744-757 (2009)

4. Guo, TL, Jiang, W: Impulsive fractional functional differential equations. Comput. Math. Appl. 64(10), 3414-3424 (2012)

5. Hernández, E, Rabelo, M, Henríquez, H: Existence of solutions for impulsive partial neutral functional differential equations. J. Math. Anal. Appl. 331, 1135-1158 (2007)

6. Henríquez, $\mathrm{H}$, de Andrade, B, Rabelo, M: Existence of almost periodic solutions for a class of abstract impulsive differential equations. ISRN Math. Anal. 2011, 1-21 (2011)

7. Hernández, E: Global solutions for abstract impulsive neutral differential equations. Math. Comput. Model. 53(1-2), 196-204 (2011)

8. Agarwal, RP, O'Regan, D: A multiplicity result for second order impulsive differential equations via the Leggett Williams fixed point theorem. Appl. Math. Comput. 161, 433-439 (2005)

9. Agarwal, RP, Benchohra, M, Hamani, S, Pinelas, S: Upper and lower solutions method for impulsive differential equations involving the Caputo fractional derivative. Mem. Differ. Equ. Math. Phys. 53, 1-12 (2011)

10. Akhmet, MU, Turan, M: The differential equation on time scales through impulsive differential equations. Nonlinear Anal. 65, 2043-2060 (2006)

11. Carl, S, Heikkilä, S: On discontinuous implicit and explicit abstract impulsive boundary value problems. Nonlinear Anal. 41, 701-723 (2000)

12. Guo, DJ: Multiple positive solutions for first order nonlinear impulsive integro-differential equations in Banach spaces. Appl. Math. Comput. 143, 233-249 (2003) 
13. Lan, HY, Huang, NJ, Kim, JK: First order nonlinear implicit impulsive integro-differential equations in Banach spaces. Dyn. Contin. Discrete Impuls. Syst., Ser. A Math. Anal. 13(6), 803-813 (2006)

14. Lan, HY: Monotone method for a system of nonlinear mixed type implicit impulsive integro-differential equations in Banach spaces. J. Comput. Appl. Math. 222(2), 531-543 (2008)

15. Lan, HY, Cui, YS: On the existence of solutions for nonlinear first-order implicit impulsive integro-differential equations. Nonlinear Anal. 71(5-6), 1670-1677 (2009)

16. Li, YX, Liu, Z: Monotone iterative technique for addressing impulsive integro-differential equations in Banach spaces. Nonlinear Anal. 66, 83-92 (2007)

17. Ahmad, B, Malar, K, Karthikeyan, K: A study of nonlocal problems of impulsive integrodifferential equations with measure of noncompactness. Adv. Differ. Equ. 2013, 205 (2013)

18. Li, J, Nieto, JJ, Shen, J: Impulsive periodic boundary value problems of first-order differential equations. J. Math. Anal. Appl. 325, 226-236 (2007)

19. Liu, LS, Wu, CX, Guo, F: A unique solution of initial value problems for first order impulsive integro-differential equations of mixed type in Banach spaces. J. Math. Anal. Appl. 275, 369-385 (2002)

20. Nieto, JJ, Rodríguez-López, R: New comparison results for impulsive integro-differential equations and applications. J. Math. Anal. Appl. 328, 1343-1368 (2007)

21. Sun, JL, Ma, YH: Initial value problems for seconder order mixed monotone type of impulsive integro-differential equations in Banach spaces. J. Math. Anal. Appl. 247, 506-516 (2000)

22. Huang, NJ, Lan, HY: Existence of the solution for a class of implicit differential equations in Banach spaces. Dyn. Contin. Discrete Impuls. Syst., Ser. A Math. Anal. 13(1), 27-36 (2006)

23. Lan, HY: Existence and uniqueness results for nonlinear first-order implicit impulsive integro-differential equations with monotone conditions. Dyn. Contin. Discrete Impuls. Syst., Ser. A Math. Anal. 17(1), 19-30 (2010)

24. Pang, $\mathrm{HH}, \mathrm{Lu}, \mathrm{M}, \mathrm{Cai}, \mathrm{C}$ : The method of upper and lower solutions to impulsive differential equations with integral boundary conditions. Adv. Differ. Equ. 2014, 183 (2014)

25. Chen, PY, Li, YX: Mixed monotone iterative technique for a class of semilinear impulsive evolution equations in Banach spaces. Nonlinear Anal. 74(11), 3578-3588 (2011)

26. Chen, $\mathrm{PY}, \mathrm{Mu}$, J: Monotone iterative method for semilinear impulsive evolution equations of mixed type in Banach spaces. Electron. J. Differ. Equ. 2010, 149 (2010)

27. Chen, PY, Li, YX, Yang, H: Perturbation method for nonlocal impulsive evolution equations. Nonlinear Anal. Hybrid Syst. 8, 22-30 (2013)

28. Li, DS: Peano's theorem for implicit differential equations. J. Math. Anal. Appl. 258, 591-616 (2001)

29. Zhang, XP, Sun, YP: Monotone iterative methods of positive solutions for fractional differential equations involving derivatives. Math. Probl. Eng. 2014, Article ID 254012 (2014)

30. Wang, F, Wang, P: Existence and uniqueness of mild solutions for a class of nonlinear fractional evolution equation. Adv. Differ. Equ. 2014, 150 (2014)

31. Pazy, A: Semigroups of Linear Operators and Applications to Partial Differential Equations. Applied Mathematical Sciences, vol. 44. Springer, New York (1983)

32. Deimling, K: Nonlinear Functional Analysis. Springer, Berlin (1985)

33. Guo, DJ, Lakshmikantham, V, Liu, XZ: Nonlinear Integral Equations in Abstract Spaces. Kluwer Academic, Dordrecht (1996)

34. Hein, HP: On the behaviour of measure of noncompactness with respect to differentiation and integration of vector-valued functions. Nonlinear Anal. 7, 1351-1371 (1983)

35. Du, Y: Fixed points of increasing operators in ordered Banach spaces and applications. Appl. Anal. 38, 1-20 (1990)

\section{Submit your manuscript to a SpringerOpen ${ }^{\ominus}$ journal and benefit from:}

- Convenient online submission

Rigorous peer review

- Immediate publication on acceptance

- Open access: articles freely available online

- High visibility within the field

- Retaining the copyright to your article 